TITLE:

\title{
Simple and longstanding adipose tissue engineering in rabbits.
}

\section{$\operatorname{AUTHOR}(\mathrm{S}):$}

Tsuji, Wakako; Inamoto, Takashi; Ito, Ran;

Morimoto, Naoki; Tabata, Yasuhiko; Toi, Masakazu

\section{CITATION:}

Tsuji, Wakako ...[et al]. Simple and longstanding adipose tissue

engineering in rabbits.. Journal of artificial organs 2013, 16(1): 110-114

\section{ISSUE DATE:}

2013-03

URL:

http://hdl.handle.net/2433/173128

\section{RIGHT:}

The final publication is available at www.springerlink.com; この論文は 出版社版でありません。引用の際には出版社版をご確認ご利用くださ $\iota_{\circ}$; This is not the published version. Please cite only the published version. 


\section{Simple and Longstanding Adipose Tissue Engineering in Rabbits}

Wakako Tsuji ${ }^{1,2}$, Takashi Inamoto ${ }^{3}$, Ran Ito $^{4}$, Naoki Morimoto ${ }^{4}$, Yasuhiko Tabata ${ }^{5}$, Masakazu Toi ${ }^{1}$

${ }^{1}$. Department of Breast Surgery, Kyoto University Hospital, Kyoto, Japan

${ }^{2}$. Adipose Stem Cell Center, University of Pittsburgh, PA, USA

${ }^{3}$. Department of Breast Surgery, Tenri Hospital, Nara, Japan

${ }^{4}$. Department of Plastic and Reconstructive Surgery, Kyoto University Hospital, Kyoto, Japan

${ }^{5}$. Department of Biomaterials, Institute for frontier medical sciences, Kyoto University, Kyoto, Japan

Key words

adipose tissue engineering, type I collagen sponge, long period, rabbits, ultrasonography

Corresponding author 
Wakako Tsuji

w-sato@kuhp.kyoto-u.ac.jp

200 Lothrop Street Pittsburgh PA 15213, USA

+1-412-383-8924 (tel)

$+1-412-648-2821$ (fax)

Second corresponding author

Masakazu Toi

toi@kuhp.kyoto-u.ac.jp

54 Kawara-cho Shogoin Sakyo-ku Kyoto, Japan

+81-75-751-3660 (tel)

+81-75-751-3616 (fax) 


\section{Abstract}

Adipose tissue engineering for breast reconstruction can be performed for patients who have undergone breast surgery. We have previously confirmed adipogenesis in mice implanted with type-I collagen sponge with controlled- release of fibroblast growth factor 2 (FGF2) and human adipose tissue-derived stem cells. However, in order to use this approach for treating breast cancer patients, FGF2 is not readily available and a large size of adipose tissue is needed. Herein, we aimed to regenerate large amounts of adipose tissue without FGF2 for a long period. Under general anesthesia, cages made of polypropylene mesh were implanted into the rabbits' bilateral fat pad. The size of cage was $10 \mathrm{~mm}$ in radius and $10 \mathrm{~mm}$ in height. Minced type-I collagen sponge was injected as a scaffold into the cage. Regenerated tissue in the cage was examined with ultrasonography, and the cages were harvested 3, 6, and 12 months after the implantation. Ultrasonography revealed a gradually increasing homogenous high echo area in the cage. Histology of the specimen was assessed with hematoxylin-eosin staining. Percentages of regenerated adipose tissue area were 76.2 $\pm 13.0 \%$ and $92.8 \pm 6.6 \%$ at 6 and 12 months after the implantation, respectively. Our results showed de novo adipogenesis 12 months after the implantation of only type-I collagen sponge inside the space. Ultrasonography is a non-invasive and useful method 
to assess the growth of the tissue inside the cage. This simple method could be a promising clinical modality in breast reconstruction. 


\section{Introduction}

Breast cancer is common disease among women worldwide. Breast surgery is not the best, but one of the local treatments of breast cancer since any defect of breast is distressing for patients. There are many ways for breast reconstruction, but each technique has its drawbacks. For example, silicone implants can induce foreign body response and be an obstacle during breast cancer screening. Autologous tissue graft implantation is a long surgical procedure, and results in operation scars at many sites. Autologous fat transfer has the drawbacks of shrinkage, fat necrosis or oil cyst formation [1]. Hence, adipose tissue engineering for breast reconstruction is a fourth alternative for patients who have undergone breast surgery.

Previous study has confirmed adipogenesis in mice implanted with a type-I collagen sponge with controlled- release of FGF2 and human adipose tissue-derived stem cells (ASCs) [2]. We showed that human ASCs not only function as progenitor cells for in vivo adipogenesis but also induce de novo adipogenesis in type I collagen scaffold. ASCs are adipose tissue-specific progenitor cells and differentiate into adipocytes or vascular cells. Type I collagen exists widely in the animal body and exhibits good biocompatibility, gelatification, and structural stability. Hence, type I collagen is a superior material for use in regenerative medicine [3, 4] [5]. FGF2 enhances 
adipogenesis [6, 7]. However, FGF2 is not readily available for breast cancer patients; furthermore, a large size of adipose tissue is needed for a long period. In addition, some diagnostic modalities are needed during follow up of the implant. The aim of this study is to regenerate large amounts of adipose tissue without FGF2 and to maintain for a long period. Another aim of this study is to evaluate the usefulness of USG as a diagnostic modality.

\section{Materials and methods}

\section{Materials}

A polypropylene (PP) mesh was purchased from FLON Industry Inc. (Tokyo, Japan). We prepared a column cage made of PP mesh with a pore size of $200 \mu \mathrm{m}$ and 5-0 prolene strings (Ethicon Inc, New Brunswick, NJ, USA). The radius of the cage was 10 $\mathrm{mm}$, and the height was $10 \mathrm{~mm}$. PELNAC, which is approved for artificial dermis, consisting of an atelocollagen sponge layer and a silicone layer was kindly supplied by Gunze Co Ltd (Kyoto, Japan). The sheet was $82 \times 60 \mathrm{~mm}$ in size. First, the silicone layer of PELNAC was removed. Then, the type I collagen layer was minced, and suspended with $8 \mathrm{~mL}$ of natural saline (Otsuka pharmaceuticals Co Ltd, Tokushima, Japan) in a 10$\mathrm{mL}$ syringe. 


\section{Animal experiments}

All the animal experiments were reviewed by the Committee on the Ethics of Animal Experiments (Faculty of Medicine, Kyoto University, Kyoto, Japan), and were performed in accordance with the Guidelines for Animal Experiments of the Faculty of Medicine, Kyoto University.

Female New Zealand white rabbits (2.5-3.0 kg; Shimizu Laboratory Supply, Kyoto, Japan) were used. General anesthesia was applied with an intramuscular injection of midazolam (4 mg/kg), medetomidine hydrochloride $(0.16 \mathrm{mg} / \mathrm{kg})$, and butorphanol tartrate $(0.4 \mathrm{mg} / \mathrm{kg})$. A PP cage was implanted into New Zealand white rabbit's bilateral fat pad, and the suspended type I collagen sponge was injected into the cage with 16G intravenous cannula. Finally, the skin was closed with a buried suture. The implant was harvested 3, 6, and 12 months after implantation, and every specimen was assessed with hematoxylin-eosin (H-E) stain. Every experimental group consisted of 4 specimens. Percentage of regenerated adipose tissue area within a cage was calculated with NIH Image J software.

\section{Ultrasonographic observation}


HITACHI MyLab25 (Hitachi medical corporation, Tokyo, Japan) was used, and ultrasonography (USG) was performed under general anesthesia at 1 week, 1 month, 3 months, 6 months, and 12 months after implantation.

\section{Statistical analysis}

Statistical differences were determined with a Wilcoxon test. A statistical significance was assigned as $* p<0.05$. JMP version 9 software (SAS Institute Inc., Cary, NC, USA) was used for statistical analysis.

\section{Results}

\section{Ultrasonographic findings}

The ultrasonographic findings are shown in Figure 2. An aechoic area and internal hyperechoic mass were noted inside the cage at 1 week (Fig $1 \mathrm{~A}$ ) and 1 month after implantation (Fig 1B). A homogenous high echo area was detected inside the cage after 3 months until 12 months (Fig $1 \mathrm{C}, \mathrm{D}$, and E).

\section{Cross section observation}

Figure $1 \mathrm{~F}$ and $1 \mathrm{G}$ show the gross sections at 3 and 6 months after implantation. At 3 
months after implantation, the appearance of the inner side the cage showed high water content (Fig 1F). At 6 months after the implantation, the appearance of the inner side of the cage became whitish compared to that of the specimen at 3 months (Fig $1 G)$.

\section{H-E staining}

Figure 1 shows $\mathrm{H}-\mathrm{E}$ staining of the specimen. At 3 months after implantation, type I collagen scaffold was observed mainly inside the cage under low magnification (Fig 1I). Under high magnification, several vacuoles were found mainly at the margins of the cage (Fig $1 \mathrm{~L}$ ). These vacuoles were morphologically adipocytes. Further, many nuclei were seen among the scaffold. At 6 months, adipose tissue occupied a large part of the cross-section surface under low magnification (Fig 1J). Under high magnification, large amount of mature adipocytes were observed and angiogenesis was noted (Fig 1M). At 12 months, mature adipose tissue was observed mostly inside the cage (Fig.1K). Complete regeneration of the adipose tissue was achieved inside the cage (Fig $1 \mathrm{~N}$ ).

\section{Percentage of regenerated adipose tissue area}

Average percentages of regenerated adipose tissue area within a cage were $31.7 \pm$ 
10.3, $76.2 \pm 13.0$, and $92.8 \pm 6.6 \%$ at 3,6 , and 12 months after implantation, respectively (Figure 2). Regenerated adipose tissue area at 6 and 12 months were significantly increased compared to at 3month after implantation $(p=0.03)$.

\section{Discussion}

Tissue engineering requires a cell source, a scaffold, and growth factors [8]. In line with these requirements, we previously confirmed adipogenesis in mice by implanting ASCs with type I collagen scaffold with a controlled- release of FGF2 [2]. Further, we confirmed in situ adipogenesis in rabbits by implanting type I collagen with the controlled- release of FGF2. Repeated administration of the mixture of collagen scaffold and gelatin microspheres containing FGF2 enabled significantly higher area at 3months after implantation than that of the single administration of each component [9]. In this study, implanting type I collagen inside the cage without ASCs and controlled- release of FGF2 resulted in adipogenesis. And an average percentage of regenerated adipose tissue within the cage was $92.8 \pm 6.6 \%$ at 12 months after implantation. Type I collagen sponge implanted into the PP cage recruited adequate cell source and necessary growth factors. Fibroblast growth factor 2 is a potent angiogenic factor, known to be effective for adipogenesis $[6,7]$. However, FGF2 cannot 
be clinically used for treating malignant diseases, because FGF2 has a potency of breast carcinogenesis [10]. Our method of adipose tissue engineering without FGF2 is a promising way to use FGF2 for clinical purposes.

Some mechanisms of adipogenesis have been reported. First, type I collagen sponge is known to be superior scaffold for ASC adhesion because of its porous structure [5] [7]. Generally, cells cannot survive in the absence of adhesive materials. So, the observation in this study that lots of nuclei with recruited cells were observed in the scaffold 3months after implantation suggested that these cells were precursors of mature adipocytes and adhered to type I collagen sponge. Collagen degradation is essential for cell migration, proliferation, and differentiation. Type I collagen scaffold have degraded and mature adipose tissue was generated inside the cage 6 months after implantation. Mature adipose tissue still remained 12 months after implantation. O'Connor et al reported a higher rate of adipogenic differentiation in vitro with type I collagen than with fibronectin [11]. We have confirmed type I collagen was excellent scaffold for adipogenesis in vivo. Secondly, the fat pad was an excellent implantation site for adipogenesis. Because the fat tissue in this study was healthy, optimal conditions for adipogenesis were satisfied. ASCs are present in the adipose tissue and are derived from the surrounding tissue. For example, administration of cell source or 
growth factors might be required for efficient adipogenesis when the fat tissue have received radiation therapy. Third, the growth factors required for adipogenesis were produced after implantation. Several growth factors are produced at the operation site after surgery. Soluble FGF2, vascular endothelial growth factor (VEGF), platelet derived growth factor (PDGF), epidermal growth factor, or insulin-like growth factor-1 (IGF-1) have been detected in the wound drainage fluid [12]. Fibroblast growth factor 2 and PDGF promote in vitro ASCs proliferation [13]. Fibroblast growth factor 2 and VEGF are potent angiogenic factors. Angiogenesis enables blood supply and oxygen to the scaffolds. Blood vessel formation was confirmed since 6 months after implantation in this study. Adipose tissue engineering requires in vivo vascularization, and in vivo vascularization enhanced the formation of engineered adipose tissue. The growth factors emanated after the surgery and contributed to adipogenesis.

The reconstruction of volume-stable adipose tissue for a long period is a great challenge. Although the subcutaneous environment is relatively static, the skin does exert a compressive force on any material placed subcutaneously. It might also be necessary to maintain some extra spaces in order to accommodate the volume-stable adipose tissue [14]. In this study, PP mesh was used to maintain this space and is a landmark of the engineered adipose tissue area. Although it is easy to detect PP mesh 
with USG, its material is unabsorbable and hard for breast reconstruction. Further studies will be needed to identify soft and absorbable materials that can be used to maintain extra space in order to apply this technique in clinical situations.

It is important to evaluate the regenerated adipose tissue. We used USG to follow up the implant over time. Regenerated tissue can be distinguished from areas with water content or empty spaces. USG is usually used for breast examination, because it is easy, non-invasive, does not involve radiation-exposure, and is cost-effective as compared to computed tomography, mammography, or magnetic resonance imaging. Herein, USG is a useful modality to assess engineered adipose tissue.

\section{Conclusion}

We achieved complete regeneration of adipose tissue inside the cage at 12 months after implantation only by implanting a type-I collagen sponge inside the space. With USG, an aechoic area and internal hyperechoic mass gradually disappeared and were replaced with homogenous high echoic area, reflecting adipogenesis. USG is a non-invasive and useful method to examine the contents inside the cage.

\section{Acknowledgement}


This work was supported by JSPS Grant-in Aid for Young Scientists (B). We thank the center for women researches in Kyoto University and Mr. Yoichi Takahashi who assisted animal experiments.

1. de Blacam C, Momoh AO, Colakoglu S, Tobias AM, Lee BT: Evaluation of clinical outcomes and aesthetic results after autologous fat grafting for contour deformities of the reconstructed breast. Plast Reconstr Surg 2011, 128(5):411e-418e.

2. Tsuji W, Inamoto T, Yamashiro H, Ueno T, Kato H, Kimura Y, Tabata Y, Toi M: Adipogenesis induced by human adipose tissue-derived stem cells. Tissue Eng Part $A$ 2009, 15(1):83-93.

3. Nakao K, Morita R, Saji Y, Ishida K, Tomita Y, Ogawa M, Saitoh M, Tomooka Y, Tsuji T: The development of a bioengineered organ germ method. Nat Methods 2007, 4(3):227-230.

4. Tanimizu N, Miyajima A, Mostov KE: Liver progenitor cells fold up a cell monolayer into a double-layered structure during tubular morphogenesis. Mol Biol Cell 2009, 20(9):2486-2494.

5. Glowacki J, Mizuno S: Collagen scaffolds for tissue engineering. Biopolymers 2008, 89(5):338-344. 
6. Kawaguchi N, Toriyama K, Nicodemou-Lena E, Inou K, Torii S, Kitagawa Y: De novo adipogenesis in mice at the site of injection of basement membrane and basic fibroblast growth factor. Proc Natl Acad Sci USA 1998, 95(3):1062-1066.

7. Kimura Y, Ozeki M, Inamoto T, Tabata Y: Adipose tissue engineering based on human preadipocytes combined with gelatin microspheres containing basic fibroblast growth factor. Biomaterials 2003, 24(14):2513-2521.

8. Langer R, Vacanti JP: Tissue engineering. Science 1993, 260(5110):920-926.

9. Kimura Y, Tsuji W, Yamashiro H, Toi M, Inamoto T, Tabata Y: In situ adipogenesis in fat tissue augmented by collagen scaffold with gelatin microspheres containing basic fibroblast growth factor. J Tissue Eng Regen Med 2010, 4(1):55-61.

10. Smith K, Fox SB, Whitehouse R, Taylor M, Greenall M, Clarke J, Harris AL: Upregulation of basic fibroblast growth factor in breast carcinoma and its relationship to vascular density, oestrogen receptor, epidermal growth factor receptor and survival. Ann Oncol 1999, 10(6):707-713.

11. O'Connor KC, Song H, Rosenzweig N, Jansen DA: Extracellular matrix substrata alter adipocyte yield and lipogenesis in primary cultures of stromal-vascular cells from human adipose. Biotechnol Lett 2003, 25(23):1967-1972.

12. Aiba-Kojima E, Tsuno NH, Inoue K, Matsumoto D, Shigeura T, Sato T, Suga H, Kato 
$\mathrm{H}$, Nagase T, Gonda K et al: Characterization of wound drainage fluids as a source of soluble factors associated with wound healing: comparison with platelet-rich plasma and potential use in cell culture. Wound Repair Regen 2007, 15(4):511-520.

13. Hauner H, Rohrig K, Petruschke T: Effects of epidermal growth factor (EGF), platelet-derived growth factor (PDGF) and fibroblast growth factor (FGF) on human adipocyte development and function. Eur J Clin Invest 1995, 25(2):90-96.

14. Cho SW, Kim SS, Rhie JW, Cho HM, Choi CY, Kim BS: Engineering of volume-stable adipose tissues. Biomaterials 2005, 26(17):3577-3585.

\section{Figure legends}

Fig 1. USG findings 1 week (A), 1 (B), 3 (C), 6 (D), and 12 months (E) after implantation. Gross views of the implant $3(F), 6(G), 12$ months (H) after implantation. H-E staining $(\mathrm{x} 4)$ at $3(\mathrm{I}), 6(\mathrm{~J})$, and 12 months $(\mathrm{K})$ after implantation. H-E staining (x20) at $3(\mathrm{~L}), 6(\mathrm{M})$, and 12 months $(\mathrm{N})$ after implantation. The scale bars in $(\mathrm{L}),(\mathrm{M})$, and $(\mathrm{N})$ indicate $500 \mu \mathrm{m}$.

Fig 2. Percentages of regenerated adipose tissue area within a cage at 3, 6 and 12 
months after implantation. Data are mean \pm standard deviation from $n=4$, statistical differences $(* p<0.05)$. 
Fig 1
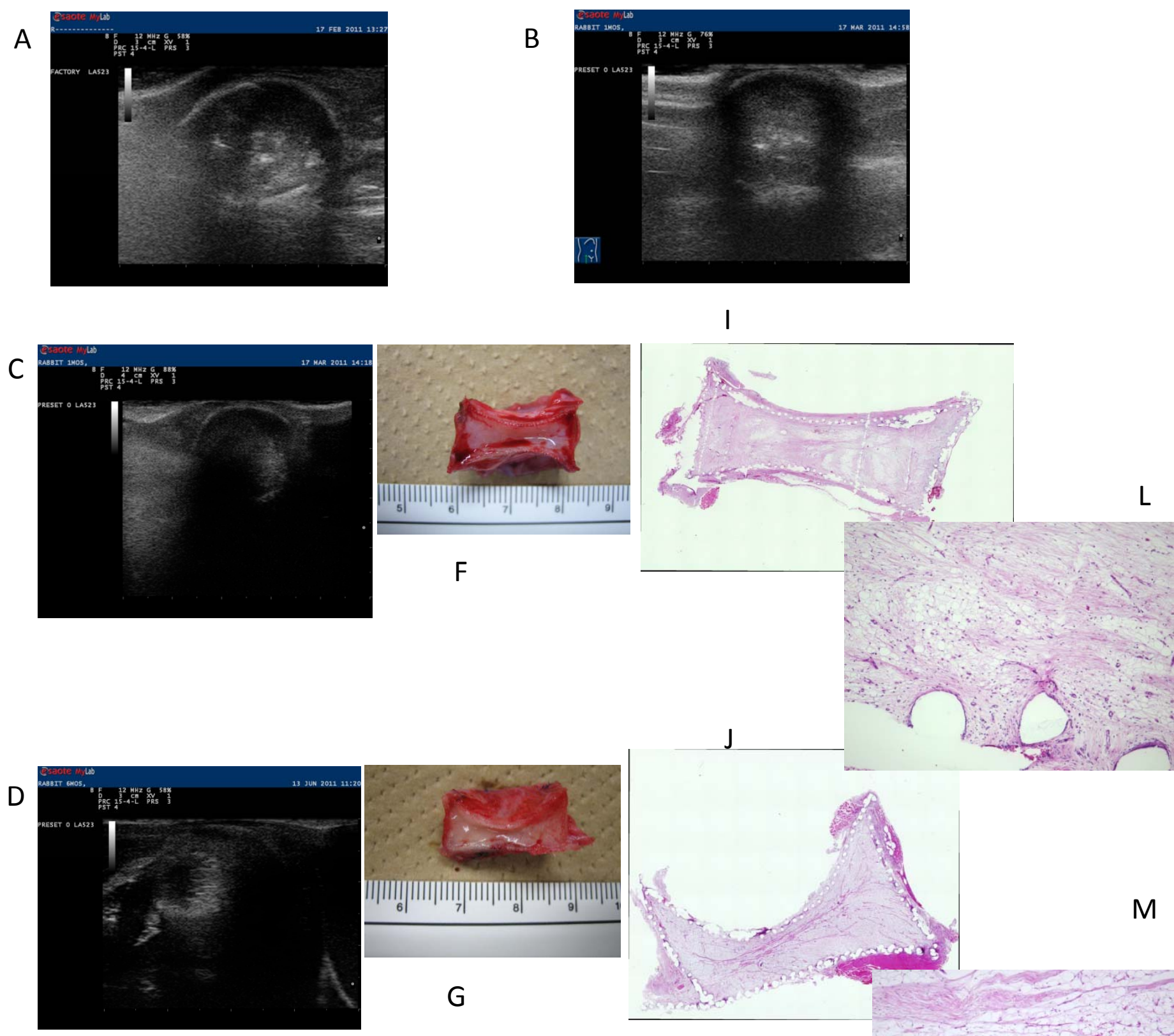

\section{G}
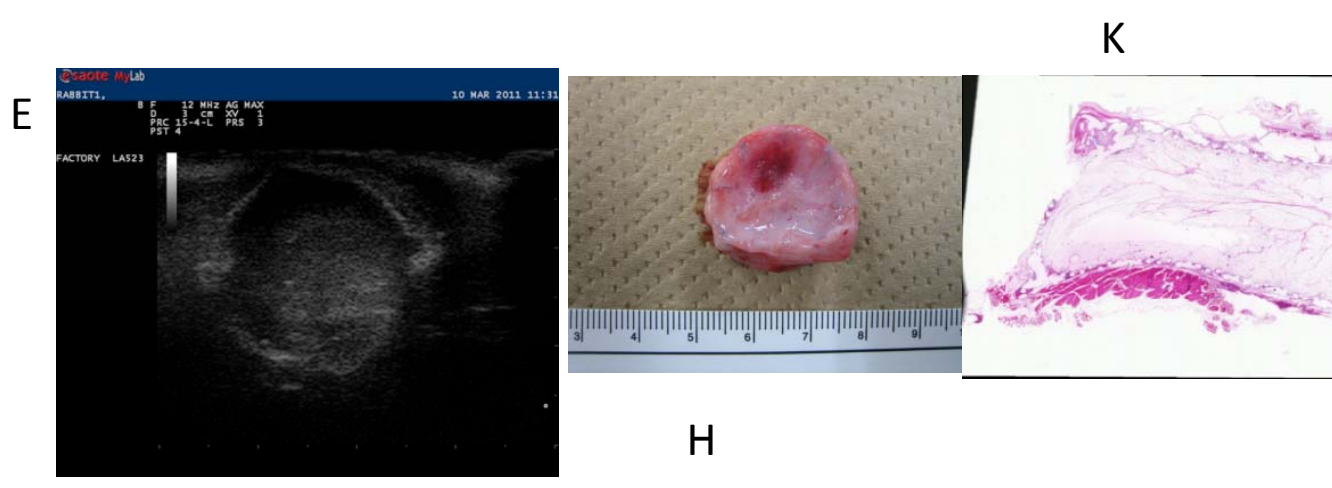

$\mathrm{H}$

○

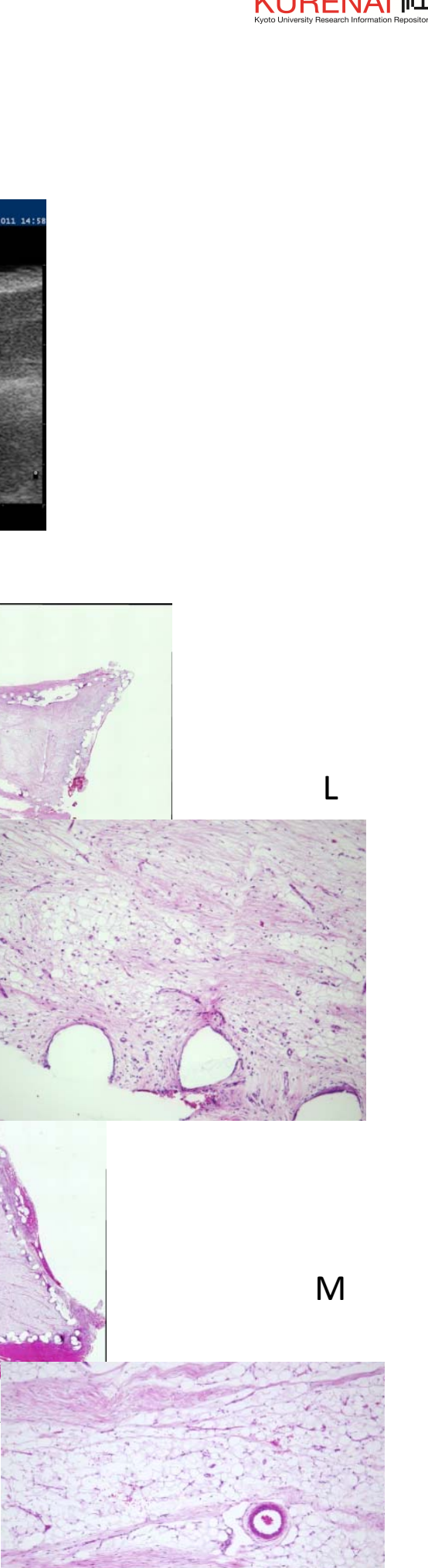

\section{F}

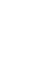

H 
Figure 2

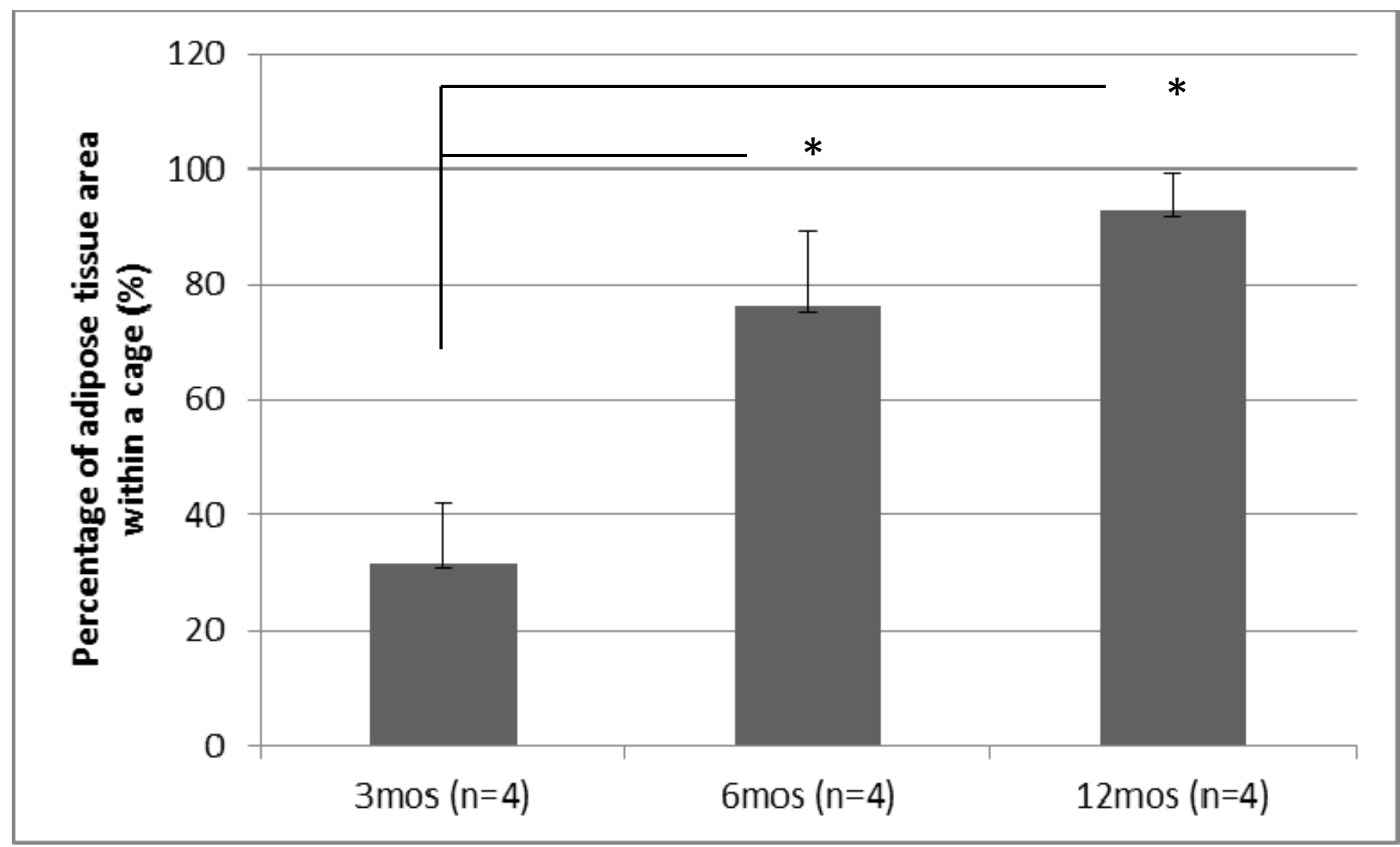

